\title{
Contributo ad uno studio sulla sismicità del Cuneese; i terremoti di Entracque del 7 Aprile 1966
}

\author{
(Contribution to a study on seismirity in the Cuneo area: \\ the earthquaties of Entrac(que on April 7, 1966)
}

\author{
M. De Pinfilis - G. Paxroccili
}

Ricevuto il 13 Jugglio 1968

\begin{abstract}
Sommario, - Il 7 Maggio 1966, il Ministero dell'Jnterno e la Prefettura di Cuneo invitavano l'Istituto Nazionale di Geofisiea a fare delle indagini su alcuni fenomeni sismici verificatisi il $7 \mathrm{Aprile} 1966$ nella Valle del Gesso, in provincia di Cuneo, o sentiti specialmente, con vivo allarme della popolazione, nella zona di Fntracque. In seguito a tale invito, grli seriventi si recarono ad lintracque e nei paesi circonvicini per esaminare sul posto grli effetti dinamici dei predet ti fenomeni e redassero poi una rela. zione, datata 30 Novembre 1966 , che, il giorno 20 del mese successivo, venne inviata alle Autorita che l'avevano richiesta.

Poiché possono costituire un contributo alle conoscenze sulle caratteristiche sismiche del Cuneese, vengono qui esposti i risultati delle indagini che vennero effettuate: oltre alla individuazione macrosismica dellepicentro, esse hamo permesso la costruzione di un sismo-cartogramma dal quale appare evidente la vasta ed irregolare propagazione del movimento tellurieo attorno all'area epicentrale. Segue un ampio esame dellattivita sismica nella media Valle del Gesso, le cui cause vengono ricercate nella tettoniea e nella costituzione geologica della zona.
\end{abstract}

Sumary. - On May 7, 1966, the National Institute of Geophysies was invited by the Ministry of the Interior and the Prefecture of cineo, to investigate some seismic phenomena which oceurred on April 7.1966 in the Gesso Valley (Province of Cuneo) and especially felt, to the great alarm of the population, in the lintracque area. Following this invitation, the authors visited Entracque and its environs to study in loeo the dynamic effects of the above mentioned phenomena. On November 30, 1966 they drew up a relation, which was forwarded to the interested Authorities on Desember 20, 1966. 
The results of said insestigation are now presented in this note as a contribution to the knowledge of the seismic characteristies of the Cuneo area: besides macroseismic determination of the epicenter, the investigations have permitted construction of a seismic map. on which the large and irresular propagation of the telluric movement around the epicentral area clearly is shown. There follows an aceurate study of seismic activity in the middle (iesso Valley, the cause of which is assumed in the tectonic and the geologre constitution of the area.

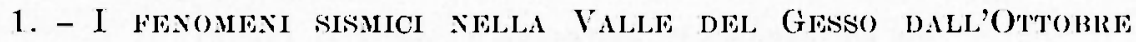 1965 MLL'O'T'OBRF 1966.}

Alle $20^{\mathrm{h}} 39^{\mathrm{m}}$ del 7 Aprile 1966 , ma scossa molto forte di terremoto, durata pochi secondi e accompagnata da un intenso boato sotterraneo, colpi la zona della media Valle del Gesso, nel Cuneese, spiegando la sua maggiore intensità (valutabile, dagli effetti dinamici, fra il VI e il VII grado della Srala Merealli) nellampia conca ove sorge il paese di Entracopue.

Circa tre quarti d'ora prima, alle $19^{\mathrm{h}} 55^{\mathrm{m}}$, una scossa premonitoria abbastanza forte (IV-V grado), accompagnata anch'essa da boato, aveva già destato un vivo allarme fra gli abitanti di quel centro montano: al sopraggiungere della scossa delle $20^{\mathrm{n}} 39^{\mathrm{m}}$, tutta la popolazione, in preda al panico, si riversava nelle strade, trascorrendo la notte all'aperto per timore che altre seosse ancora più forti potessero provocare dei crolli. Per effetto del movimento sismico rimasero lesionati, non gravemente, l'edilicio delle scuole elementari, quello dell'ospedale e numerosi altri fabbricati, per lo più vecohi e di difettosa costruzione. In molti edifiei si aggravarono sensibilmente lesioni preesistenti, probabilmente dovute alla sovrapposizione degli efletti di antichi movimenti tellurici nella Valle del Gesso, la quale è dotata di una sua propria, sia pur non elevata sismicità. Aleuni giorni dopo l'avvenimento, una dozzina di abitazioni, giudicate pericolanti dai tecnici del Genio Civile di Cumeo, vennero fatte evacuare dalle autorità commuali.

A Valdieri, il maggior centro della Valle del Gesso, situato, in linea d'aria, circa quattro chilometri a Nord di Entracque, le scosse si manifestarono con un'intensità di poco inferiore. La seconda, sentita anche là con spavento generale e fuga dalle case, vi laggiunse il grado VI, causando screpolature negli intonachi a fenditure nei muri di aleuni vecchi fabbricati. 
Alle due scosse predette fecero seguito, nella stessa giomata. tre repliche di mediocre intensita: alle $21^{\mathrm{h}} 08^{\mathrm{m}}$, alle $22^{\mathrm{h}}+8^{\mathrm{m}}$ e alle $20 \mathrm{n} 5 \mathrm{~m}$ lispettivamente. Durante il giorno segruente, sei deboli scosse, quattro nella mattinata e due nel pomeriggio, furomo avvertite nella sola zona di Entracque. Altre due, anchesse lievi, si ebbero nelle prine ore del 9 Aprile. Sucoessivamente, per quasi tutto il mese di Aprile, leggieri e frequenti moti sismici, spesso aceompagnati da lombi, e, talom, rombi senza scosse percepite dall' nomo vemnero sentiti nella sola zona di Entracque e, più ramamente, anche in quella di Valdieri.

Lievi scosse e rombi vemnero di quando in quando arvertiti, sebbene con frequenza molto minore, anche durante il mese di Magrgio.

In seguito, le manifestazioni sismiche percepite dalle persone nella zona di Entracoue divemnero sempre più rare. Secondo informazioni ricevute da persone colà residenti, poche, leggiere o leggerissime, furono le scosse avvertite nel mese di Giugno; soltanto due, leggiere, nel mese di Lugrio (21 $20^{\mathrm{m}}$ del giorno 22 e $01^{\mathrm{h}}$ del giorno 28 ); nessuma nel mese di Agosto; ma infine, mediocre, in Ottobre $\left(02^{\mathrm{h}} 09^{\mathrm{m}}\right.$ del gromo 10). Da allora in poi nessun movimento tellurico vemne più avvertito dalla popolazione.

Per completare il quadro dei sopra esposti avvenimenti sismici nella Valle del Gesso, ocoore dire che i terremoti del 7 Aprile non giunsero all'improvviso, ma furomo preceduti da numerose manifestazioni sismiche minoli, sicchó oggi essi possono essere considerati come la fase principale di $u n$ lungo periodo di attivita sismica. Tale attivita comincio ad essere notata nellottobre del 1965, continuo sin alla line del maryo 1966 con gruploi di scosse d’intensiti modesta, ma di frequenza a volte rilevante (*), separati da pause più o meno lunghe; ebbe $u n$ massimo nel sisma delle $20^{\mathrm{h}} 39^{\mathrm{m}}$ del $T$ Aprile $1966 \mathrm{e}$, come si è detto, si protrasse con scosse sempre meno frequenti e d"intensità in generale molto leggiera lino all'ottobre del 1966.

Lo stato di apprensione suscitato negli abitanti dei Comuni di Valdieri e specialmente di Entracque dal prolungansi dei fenomeni sismici cominciati nell'Ottobre del 1965 divenne vivissimo dopo quelli del 7 Aprile 1966 e tale si mantenne per tutto il successivo mese di Maggio.

(*) Nella prima metir di Genmaio. ach esempio, secondo un elenco fornitoci dal Genio Civile di Cumeo, furono avvertite ad Entrac(ue ben 30 scosse tutte dintensita non superiore al IV grado e precisamente: 4 di grado II. 22 di III. 3 di III-IV a ma di IV. 
2. - EpICENTRO F PROPAGHZIONE MACROSISMICA DEL TERREMGTO DEL 7 Aprite $1966\left(20^{\mathrm{h}} 39^{\mathrm{m}}\right)$.

Per portare a compimento l'incarico ricevuto, il 25 Maggio 1966 gli scriventi si recarono a Cuneo e di là, dal 26 al 29 Maggio, portandosi nelle varie località mediante un automezzo messo a disjosizione dalla locale Prefettura, effettuarono accurate indagini macrosismiche sul terremoto del i Aprile 1966, nell'intento di individuare la posizione approssimata dell'epicentro e di chiarire le modalità di propagazione intorno alla zona epicentrale.

Venne fatta anzitutto, insieme ad alcuni membri dell'Amministrazione commale, una ricognizione nellabitato di Entracque, ove erano stati lamentati più sensibili damni e dove più vivo era stato l'allarme della popolazione. In base agli eftetti riscontrati sugli edifici (efletti già esposti in sintesi nel paragrafo precedente), è da ritenere, come già si è detto, che la scossa delle $20^{\mathrm{h}} 39^{\mathrm{m}}$ del $T$ Aprile abbia raggiunto nella conca di Entracque un'intensita compresa fra il VI e il VII grado della Scala Mercalli. Con uguale intensiti vemne colpita la frazione S. Iucia, che dista circa $2 \mathrm{~km}$ dal capoluogo in direzione $\mathrm{SE}$, e con intensita un po' minore (VI grado) la frazione Triniti situata nel Vallone del Torrente Bousset, affluente del Gesso, circa $\downarrow \mathrm{km}$ a SE di Entracque.

Con la cortese guida di funzionari del Compartimento di Torino dell'ENEL, furono visitate anche le opere dell'impianto idroelettrico Piastra-Andonno: in particolare l'imponente diga, detta della Piastra, costruita circa un chilometro e mezzo a monte di Entracque. Questa diga, a gravità in conglomerato rementizio, lunga al coronamento 420 metri el avente un'altezza massima di 88 metri sulle fondazioni, crea un lago artificiale della capaciti di 12 milioni di metri cubi, il quale si estende per km 1,8 lungo la Valle del Gesso di Entracque e raccoglie, direttamente e mediante allacciamenti, le acque di diversi rami ed affluenti del Gesso ntilizzando un bacino imbrifero di circa 245 chilometri quadrati. Una galleria in pressione, con un percorso di circa dodici chilometri entro le formazioni calcareo-dolomitiche dei contrafforti settentrionali dei Monti Bussaia e Sape, collega il lago artificiale della Piastra con una centrale idroelettrica situata in prossiniti di Andomo (Tav. 1). La diga, impostata su saldissime rocce gneissiche non risenti il minimo damno dai movimenti tellurici. 


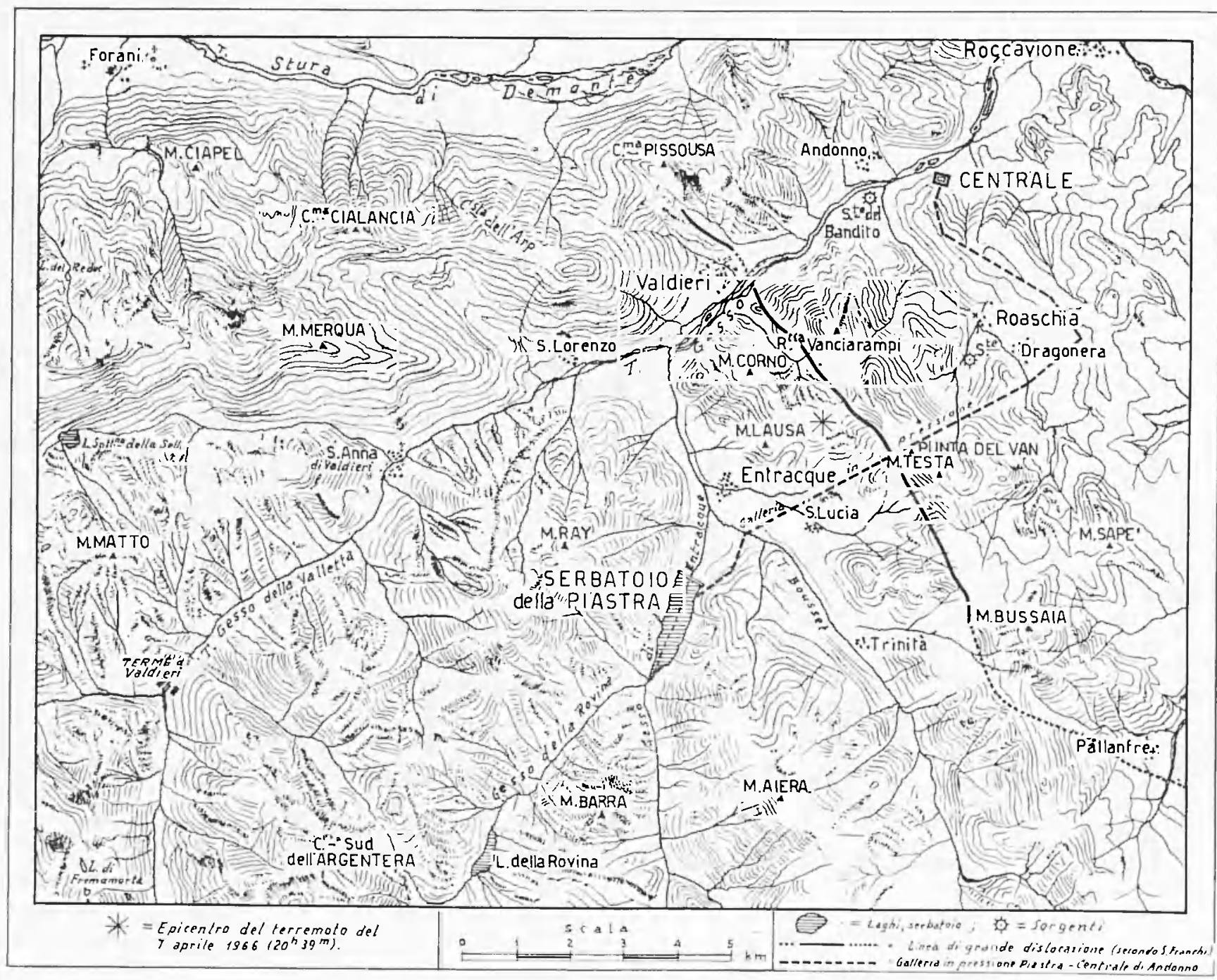


Oltre che nella conca di Entracque, furono compinti sopralluoghi in altri centri della Valle del Gesso: a Valdieri, Andomno, S. Anma di Valdieri, Terme di Valdiori e a Roaschia, nel vallone omonimo. Nella prima delle menzionate localita, la scossa fu sentita molto fortemente (VI grado); un po' meno a Roaschia (V-VI); ad Andomo, fortenente (V); a S. Anna mediocremente (IV); leggermente alle Terme di Valdieri (III).

Furono poi visitati tutti i centri della Valle Vermenagna lino al Colle di Tenda e quelli della Val Gunde lino a Palanfoc. In questa località, situata sul versante Sud-Orientale del Monte Bussaia, a 1379 metri di altitudine, la scossa fu alquanto energica (V-VI); a Tetto Folchi, pure nella Val Grande, a quota 1025, un po' meno (V).

Successivamente, i sopmalluoghi furono estesi ai rentri della Valle Stura di Demonte, della Val Grana e della Val Maira. Vennero effettuate inclagini anche in alcune localita della pianuma in prossimita di c'uneo.

I dati macrosismici ottennti nei numerosi paesi visitati e quelli ricavati da appositi questionari inviati a molti commi delle province di Cuneo, Torino, Asti, Imperia e Savona (*) hamo permesso la compilazione dell'unito sismo-cartogramma (Tav. 2). I'epicentro maceosismico, in base alla distribuzione delle intensità nei luoghi piu prossimi all'area maggiormente colpita, sembra potersi porre, con buona approssimazione, nei pressi del punto di coordinate geografiche $44^{\circ} 15^{\prime} \mathrm{X}$ e 7025' E, cioce vicino alla Punta del Van, ad una distanza di cirea due chilometri, in direzione XE, dallabitato di Entracque (Tav. 1).

Come appare dalla Tav. 2, la zona racchiusa dalla isosista di grado VI ha una forma pressoche ellittica con l'asse maggiore in direzione NNW-SSE e della lungheza di ma quindicina di chilometri. Entro questa zona, eccontricamente, si trova la conca di Entracque, dove la scossa raggiunse un'intensità maggiore (VI-VII) e che possiamo pertanto considerare come l'area epicentrale.

I'isosista di grado $V$ segue abbastanza fedelmente l'andamento di quella di grado VI e comprende per intero la Valle Vermenagna,

(*) Isa Prefetura di Cuneo ha cortesemente collaborato con noi assumendosi l'incarico di distribuire, secondo le nostre indicazioni, i questionari macrosismici e curandone successivamente la raccolta. Tutti i Comuni i cui nomi figurano nella Tav. 2 sono stati interpellati ed hamo risposto alla nostra inchiesta. 


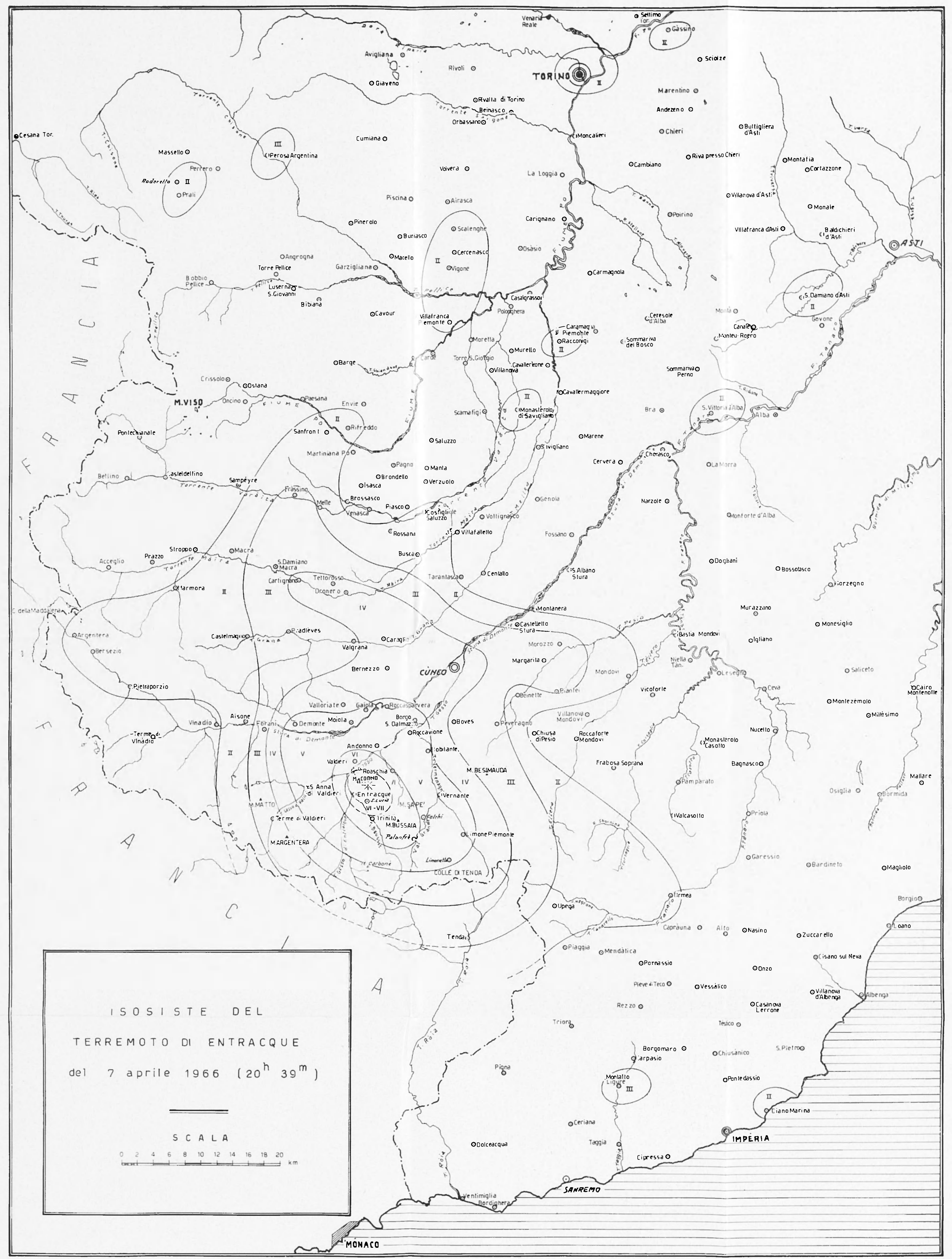


quasi per intero la Valle del Gesso con le sue varie ramificazioni e all'incirca da Roceasparvera a Demonte la Valle della Stura.

Il tracciato delle isosiste di grado inferiore è invece molto irregolare. Si noti, per esempio, come la scossa, sentita fortemente a Demonte, sia stata avvertita solo leggermente nella vicina località di Forani e sia passata completamente inosservata nella pur vicina Vinadio. Altre anomalie risultano dall'andamento sinuoso dell'isosista di IV grado: l'intensità, di grado V a Moiola, in Val di Stura, discende bruscamente fino a Valloriate (IV), pure in Val di Stura, decresce ancora, ma più lentamente, fino a Valgrana (III), nella vallata omonima, per tornare a crescere a Dronero e a Cartignano (IV), nella Val Maira.

Benché l'intensità epicentrale del terremoto non abbia raggiunto un valore molto elevato, pur tuttavia la propagazione della scossa risultò assai estesa, poiché essa venne percepita dalle persone anche a notevoli distanze dall'epicentro. Anche sotto questo riguardo però, come appare dalla Tav. 2, si ebbero numerose anomalie: verso Nord, ad esempio, la scossa fu avvertita a Torino e a Gàssino Torinese, cioè ad una distanza epicentrale di un centinaio di chilometri, e non lo fu invece affatto in tante località nella stessa direzione, ma molto più vicine all'epicentro. Esternamente alla zona limitata dalla isosista di grado II, la scossa venne inoltre avvertita, con lieve intensità, nei Comuni di Prali, Perosa Argentina, Scalenghe, Cercenasco, Vigone e Villafranca Piemonte, tutti della provincia di Torino; nei Comuni di Racconigi, Monasterolo di Savigliano e S. Vittoria d'Alba, della provincia di Cuneo; a S. Damiano in provincia di Asti; a Montalto Ligure e a Diano Marina, in provincia d'Imperia.

Verso Sud, secondo notizie apprese alla Galleria del Col di Tenda dai gendarmi francesi, la scossa sarebbe stata avvertita di III grado nella cittadina di Tenda e, secondo informazioni comunicate dal "Bureau Central International de Séismologie », pure di III grado a Monaco.

Sotto la forma microsismica il movimento fu registrato in alcuni Osservatori italiani, fra i quali Salò e Trieste, e in parecchi stranieri (Strasburgo, Isola, Monaco, Praga, ecc.).

La irregolare propagazione macrosismica unitamente alla grande estensione dell'area interessata dal terremoto fanno ritenere che esso debba aver avuto una notevole profondità ipocentrale. 


\section{3. - La sismicita della VAlLe del Gesso.}

Il Cuneese è una zona sismica caratterizzata da numerosi centri di scuotimento tellurico, i quali, per la magrgior parte, sono situati nelle valli alpine che convergono verso l'estremità Sud-Occidentale della Pianura Padana, ove si estende la città di Cuneo, cioè: la Val Maira, la Valle Stura, la Valle Vermenagna e la Valle del Gesso. Nonostante la notevole frequenza dei terremoti dovuti ai centri anzidetti, la sismicità del Cuneese può ritenersi in complesso piuttosto modesta poiché le intensità dei sismi corocentrici raramente hanno raggiunto il grado VII, eccezionalmente il grado VII-VIII (*) e mai, almeno in tempi storici, un valore maggiore; e anche i terremoti esocentrici, con origine nella Liguria Occidentale, nel Pinerolese e nelle Alpi francesi, che talora si propagarono fino alle vallate del Cuneese, mai vi giunsero con intensità superiori al grado VII.

Poco sopra il suo sbocco in pianura, la Valle del Gesso si ramifica in parecchie valli secondarie nell'ambito delle quali si ergono le cime più elevate delle Alpi Marittime, tutte dominate dall'imponente massiccio gneissico dell'Argentera. La parte inferiore e quelia media della valle, fin poco a monte di Entracque e di S. Lorenzo di Valdieri, sono costituite prevalentemente da potenti formazioni calcaree di età mesozoiche ed è appunto in tali parti che hamno generalmente avuto origine, anche nei secoli passati, i movimenti tellurici della Valle del Gesso. La parte superiore, che con i suoi vari rami si svolge entro le rocee cristalline antiche dell'Argentera, è praticamente asismica.

Due sono i centri di sismicità accertati nella Valle del Gesso: l'umo allo sbocco della valle, in prossimità di Roccavione, e l'altro nella zona di Entracque-Valdieri.

Sulla base delle ricerche di G. Mercalli del 1897 e dei dati rilevati posteriormente da $A$. Cavasino e J. P. Rothé, si può affermare che le scosse telluriche attribuibili al centro di Roccavione non hanno mai raggiunto, in tempi recenti, intensità superiori al grado VI; alcune di esse però, come per esempio quelle del 20 Aprile 1901 e 30 Magrio

$\left({ }^{*}\right)$ Tale intensità raggiunsero forse i terremoti seguenti : 23 Marzo 1835 , con epicentro nei dintorni di Boves, in prossimità dello sboceo della Valle Vermenagna; 30 Agosto 1858, con epicentro nella bassa Valle della Stura, in prossimitì di Demonte; 7 Giugno 1878, con epicentro nella bassa Valle Maira, nei pressi di Cartignano. 
1905, sentite di VI grado a Roccavione, furono caratterizzate da una propagazione macrosismica molto vasta ed irregolare.

L'elenco delle scosse sismiche ch'ebbero origine nella zona di Entracque-Valdieri ̀̀ di gran lunga pì̀ esteso di quello relativo al centro di Roccavione. Tali scosse però, ove si eccettui quella del 7 Aprile 1966, furono sempre di intensità assai modesta (inferiore al grado VI) e di estensione macrosismica limitatissima: i relativi ipocentri dovettero essere quindi di profondità molto piccola. Esse inoltre assai raramente si presentarono raggruppate in periodi sismici e quando ciò avvenne si trattò quasi sempre di periodi brevi. Il recente periodo sismico culminato nella scossa del $\tau$ Aprile rappresenta quindi, per la durata ed anche per l'estensione macrosismica della scossa di maggiore intensità, un evento piuttosto singolare. Per trovare un altro periodo abbastanza lungo, d'intensità però leggera, bisogna risalire al 1887. Il 4 Marzo di quell'anno, cioè pochi griorni dopo il grande terremoto ligure del 23 Febbraio 1887 , dal quale tutto il Cuneese fu colpito con fortissima intensità, ebbe inizio nella conca di Valdieri, con una scossa di grado III, un periodo sismico che, con scosse sempre assai lievi, si prolungò poi per circa due mesi.

A complemento di quanto sopra esposto, si deve aggiungere che assai spesso nella Valle del Gesso si sono propagati, mai però con intensità molto forte, i terremoti provenienti dai centri sismici delle altre valli cuneesi. Di tali centri daremo qui un rapido cenno.

Due ne esistono nella Val Maira: l'uno fra Dronero e Cartignano, la cui maggiore manifestazione, a tuttoggi, resta la scossa fortissima del 7 Gingno 1878 (v. nota a pag. 332), che ebbe una propagazione assai ampia ed irregolare; l'altro, verso la testata della valle, in prossimità di Prazzo. Quest'ultimo, che fino a pochi decenni fa poteva essere considerato del tutto secondario per le sue rare e deboli manifestazioni, ha dato origine in questi ultimi tempi a terremoti abbastanza importanti, caratterizzati da propagazioni macrosismiche molto irregolari e di notevole vastità. Ricorderemo la scossa di grado $V$ del 9 Luglio $1936\left(^{*}\right)$ e quella fortissima (grado VII) del 20 Giugno 1955 (epic. macrosismico $44^{\circ} 29^{\prime} \mathrm{N}$ e $\left.7^{\circ} 05^{\prime} \mathrm{E}\right)\left({ }^{*} *\right.$, ambedue sentite nella zona di Entracque-Valdieri, la prima di III e la seconda di IV grado.

(*) L'epicentro di questa scossa, seconclo Rothé, è nel punto di coordinate $44^{\circ} 26^{\prime} \mathrm{X}$ e $7^{\circ} 00^{\prime} \mathrm{E}$, cioc̀ circa sei $\mathrm{km}$ a SW di Prazzo.

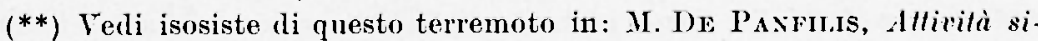
smica in Italia dal 1953 al 1957, "Annali di Geofisica ", XII, n. 1, 1959. 
Tel bacino dellampia Valle della stura di Demonte, con la quale confina la Valle del Gesso, sono molto probabilmente due focolari sismici: l'mo in prossinità di Demonte e l'altro fra Vinadio e Bagni di Vinadio. I sismi irmadiati dal primo, eccetto pochi casi (p. es. la scossa del 30 Agosto 1858 che fu di VI-VII grado), sono stati d'intensità modesta e di carattere locale. Dal secondo hanno tratio origine numerose scosse anch'esse però quasi tutte d’intensità non elevata e molto localizzate. Qui giova citare, fra esse, la scossa del 2 Gennaio 1893 e quella del 19 Febbraio 1935. Ia prima ebbe l'intensità di V-VI grado a Bagni di Vinadio e fu avvertita di IV-V in Valle Gesso (Valdieri, Entracque, Roaschia); la seconda raggiunse, come intensità massima, solo il grado $\mathrm{V}$, ma ebbe una notevole estensione (*).

A Sud di Cumeo, la Valle che prende il nome dal Torrente Vermenagna, principale affluente del Gesso, è una zona di vivace attività sismica. L'esame dei terremoti in essa avvenuti porta ad ammettere l'esistenza di un centro di scuotimento fra Vermante e il Colle di Tenda. Anche questi sismi, che di rado superarono il grado VI, si propagarono qualche volta sensibilmente nella Valle del Gesso. Ricorderemo qui il terremoto del $1^{\circ}$ Luglio 1885 , di VI-VII a Vernante, sentito fortemente a Entracque, Roaschia e Valdieri e quello del 26 Novembre 1892, di VI grado a Vernante e sentito di IV - V nella media Valle del Gesso.

Chiuderemo questa sintesi delle manifestazioni sisniche nella Valle del Gesso rammentando il terremoto avvenuto nel Cuneese alle $11^{\mathrm{h}} 5^{\mathrm{m}}$ del 4 Maggio 1958, notevole non tanto per la sua massima intensita (VI grado), quanto per la vastiti della sua propagazione. I'area mesosismica, dove la scossa raggiunse il grado VI, ha all'incirca la forma di un'ellisse con l'asse maggiore, della lunghezza di una ventina di chilometri, estendentesi dalla bassa Valle del Gesso allo sbocco della Val Grana e l'asse minore di circa 10 chilometri (Tav. 3). A Caraglio, Rittana, Gaiola, Borgo S. Dalmazzo, Roccavione, Valdieri, Andommo, località contenute nell'area predetta, la scossa fu da tutti sentita molto fortemente accompagnata da rombi; si ebbero cadute di calcinacci e lievi fenditure in diverse case. Il centro approssimativo di tale area

${ }^{*}$ ) Allepicentro di questo sisma J. P. Rothé assegna le coordinate $44^{\circ} 15^{\prime} \mathrm{N}$ e $7^{\circ} 05^{\prime} \mathrm{E}$. Esso pertanto cadrebbe pochi chilometri a sud di Bagni di Vinadio. 


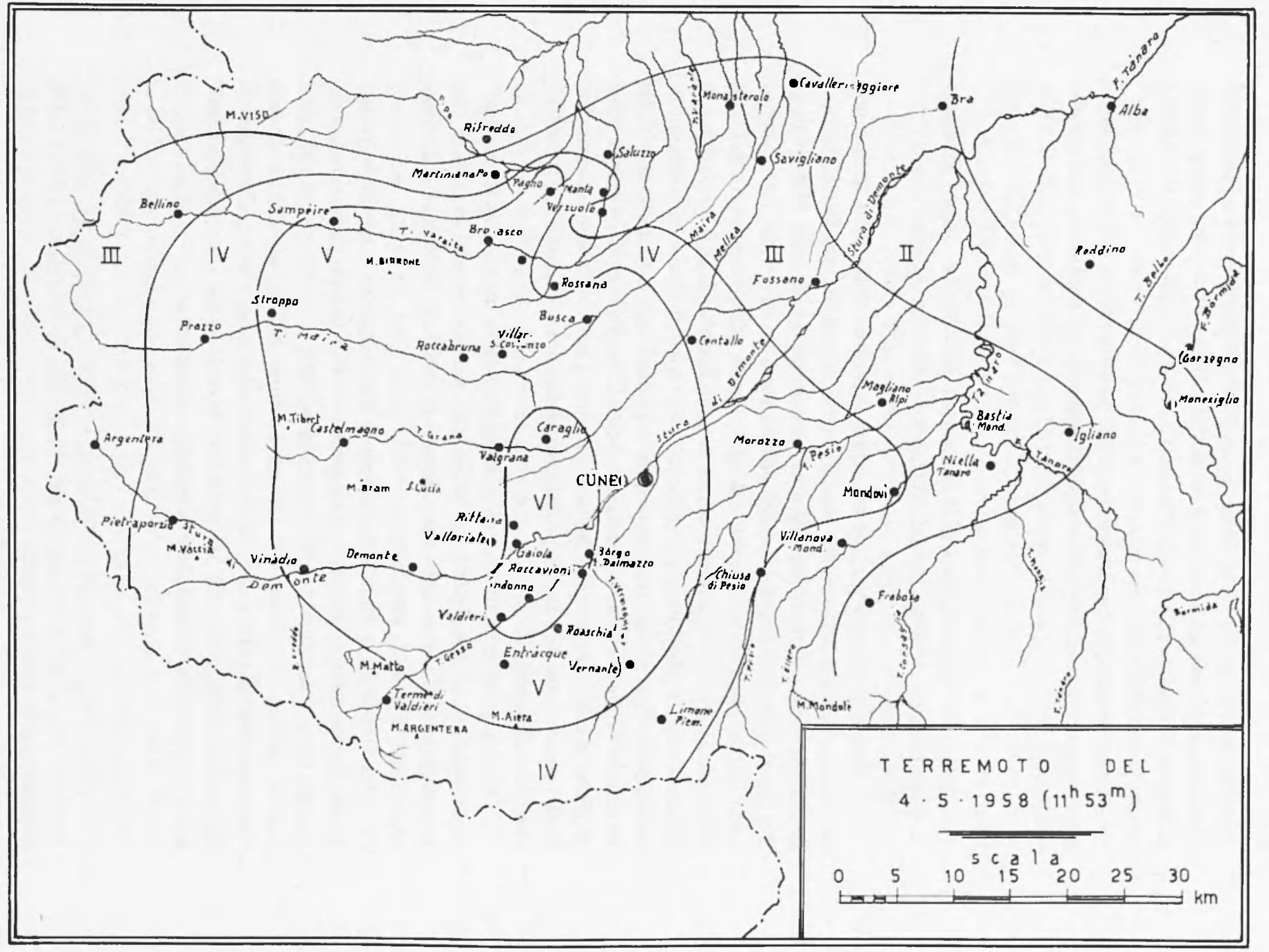


$\left(44^{\circ} 20^{\prime} x, 7^{\circ} 24^{\prime} \mathrm{E}\right)$ cade nella bassa Valle della Stura in prossimità di Gaiola e vicino ad una linea di grande dislocazione che corre lungo il fianco sinistro della valle medesima. Fella conca di Entracque la scossa fu sentita di $V$ grado.

Cna replica di mediocre intensità avvenuta il giorno 13 dello stesso mese, venne avvertita leggermente nella media e bassa Talle del Gesso.

\section{4. - Ceñi sUla COSTITUZTONF geOLOGICA DEILA ZONA ENTRAC- QUESE (*) - POSSIBIJI CAISE DFI TERREMOTI COROCENTRICI.}

Il paese di Entracque, ad una quota di circa 900 metri, si estende entro una vasta e bella conca racchiusa fra le pendici del Monte Lausa, propaggine meridionale del Monte Corno, e quelle dei Monti Ray, Testa ed liera (Tav. 1). I suoi edifici sono costruiti in parte sopra una coltre alluvionale depositata dal Gesso d'Entracque e dal suo affuente Bousset ed in parte sopra piccoli lembi calcarei che affiorano nella ronca in direzione SE-NO. Questa costituzione del terreno di fondazione contribui probabilmente a rendere più sensibili gli effetti meccanici della scossa del $i$ Aprile poiché, com'è noto, i terreni alluvionali, già di per se stessi pericolosi sotto l'urto delle onde sismiche, lo divengono ancor più in prossimità delle linee di contatto con terreni più compatti.

Lungo i margini della conca, i fianchi delle montagne circostanti sono ammantati da detriti di falda e da estesi depositi morenici che testimoniano l'imponente glacialismo di cui fu sede la Valle del Gesso nel Periodo Diluviale.

Ad Ovest ad a Sud della conca d'Entracque, si ergono le imponenti formazioni gneissiche paleozoiche del Gruppo montuoso dell'Argentera, culminante nella cima omonima a 3297 metri di altitudine. Quasi al centro del Gruppo, affiomano estesamente i graniti che costituiscono il nucleo del massiccio cristallino. Non si ha alcuna notizia di terremoti con epicentro in questa zona, la quale, attualmente, può essere considerata quasi asismica.

$\left(^{*}\right)$ V.i fogli "Boves" e "Demonte " della Carta geologiea ditalia rilevati, il primo, da D. Zaccagna con il concorso di S. Franchi (1934), ed il secondo da S. Franchi (1933). 
Il Massiccio cristallino dell'Argentera, che alcuni autori sostengono essere di etì paleozoica nella sua parte superiore ed arcaica in quella inferiore, è circondato da potenti formazioni sedimentarie mesozoiche e cenozoiche. Nei grandiosi movimenti dellorogenesi alpina susseguitisi dall'Eocene in poi, tali formazioni, potentemente compresse contro la massa gneissica del Massiccio, si corrugarono ripetutamente e, sotto l'azione di pressioni enormi, vennero contorte, fratturate a variamente dislocate le une rispetto alle altre.

A Nord-Est del Massiccio, nella zona entracquese, si addossa agli gneiss di Monte Ray e del Gruppo del Monte Aiera una vasta e potente formazione calcarea del Cretaceo, culminante, nella sua parte settentrionale, con la cima del Monte Corno (m 1506). In essa sono incise la conca d'Entracque e la breve valle che unisce la conca medesima con quella di Valdieri.

Ad Est di detta formazione cretacea, separata da essa da terreni fossiliferi eocenici, affiora un'altra grande formazione calcarea, ma di età giurassica, che dal Monte Bussaia (m. 2451), attraverso la Punta del Van (m. 1761), giunge fino alla Rocca Vanciarampi. Fra questa formazione ginrassica e i predetti terreni eocenici passa una linea di grande dislocazione, la quale (secondo i fogli "Boves" e "Demonte " della Carta geologica d'Italia) ha inizio dal Colle di Tenda, attraversa l'impervia zona del Monte Bussaia, poi, dopo un percorso quasi parallelo alla Valle del Bousset, passa per la Punta del Van, circa $3 \mathrm{~km}$ ad ENE di Entracque e si prolunga infine, al di là del Gesso di Valdieri, verso la Valle della Stura di Demonte.

L'epicentro del terremoto de] 7 Aprile 1966, secondo la individuazione macrosismica indicata precedentemente, cade ad ovest ed a brevissima distanza dalla linea tettonica predetta. Esso è situato entro una fascia sismica, messa in evidenza da J. P. Rothé e da lui chiamata "arco sismico piemontese ", che dal Col di Tenda, seguendo i] limite orientale delle Alpi lungo la Pianura Padana, giunge fino a Sud del Iago Maggiore.

Con il grande sviluppo delle formazioni calcaree nella media Falle del Gesso è da porre in relazione la circolazione carsica assai intensa delle acque sotterranee, della cui esistenza sono prova evidente le numerose abbondanti sorgenti che esistono qua e lì nella zona, come per esempio le scaturigini del "Bandito" e di "Cialombard" sulla sponda destra del Gesso di Valdieri e la sorgente "Dragonera " nel Vallone di Roaschia. Come conseguenza delle azioni di erosione chimica e meccanica esercitate per secoli e secoli dalle acque sotter- 
ranee, ì da ritenere che molto sviluppata debba essere la cavernosità del sottosmolo. Te è una prova la "Gaverna del Bandito" situata sulla riva destra del Gesso di Valdieri poco a monte delle sorgenti omonime sopra citate, ben nota anche per numerosi rinvenimenti di resti ossei di "Crsus speloeus"; ne sono prova altresì altre caverne, non peró della importanza di quella del Bandito, di cui fa menzione il geologo F. Sacco in ma nota del 1890: fra esse, molte situate in prossimità del paese di Roaschia e alcune nella Val Grande di Vernante, in prossimità di Palanfrè.

La sismicità della media Valle del Gesso è con grande probabilità una conseguenza naturale delle condizioni geologiche sopra accennate.

I terremoti di gran lunga più frequenti nelle Talli cumeesi, in quella del Gesso in particolare, sono stati fino ad oggi i terremoti locali, cioè quelli caratterizzati da una superficie macrosismica assai ristretta e, quindi, da una profondità ipocentrale molto piccola (forse, in molti (asi, non superiore a un km). Ia loro intensità è stata sempre modesta e raramente ha raggiunto il VI grado della Scala Mercalli. E probabile che le scosse sismiche di questo tipo, almeno alcune, traggano la loro origine da scoscendimenti bruschi e da successivi assestamenti nell'interno delle masse calcaree, già fratturate in vari blocchi per effetto di movimenti orogenetici e poi grandemente erose dall'ingente circolazione di acque sotterranee.

Molto diversi, sia per l'intensità sia soprattutto per la profondità, appaiono gli aspetti della scossa principale del 7 Aprile 1966. Come già si è detto, la sua irregolare propagazione e il fatto che essa, pur non avendo raggiunto un'intensità molto elevata, sia stata percepita dall'nomo anche in località distanti un centinaio di chilometri dall'epicentro e registrata dagli strumenti in Osservatori distanti centinaia di chilometri, sono non dubbi indizi di ma profondità ipocentrale pinttosto accentuata. L'orogenesi nelle Alpi non si è ancora conchiusa e la tettonica non ha ivi ancora raggiunto il suo assetto definitivo: le scosse sismiche del 7 Aprile 1966, quelle sopra ricordate del Maggio 1958 e le altre di caratteristiche simili che in varie epoche hamno colpito le Valli del Cuneese sono probabilmente da ascrivere a brusche dislocazioni di strati profondi della crosta terrestre sottoposti all'azione di enormi tensioni derivanti dall'orogenesi ancora in atto.

Nel chindere la relazione di cui è stato fatto cenno nel Sommario, gli scriventi auspicavano, per un approfondito studio della sismiciti del Cuneese, ed in special modo della Valle del Gesso ove esistono importanti impianti idroelettrici, la istituzione di una rete di sismografi 
di tipo adatto ed opportumamente situati nella zona. Sono lieti pertanto di avere appreso che, a cura dell'Istituto Geofisico e Geodetico dell'Universita di Genova, per incarico dato dal Compartimento di Torino dell'EXEL, è stata installata nel Cuneese, ed ̀̀ attualmente in funzione, una rete di tre stazioni sismiche ubicate, rispettivamente, a:

S. Anna di Valdieri $\left(44^{\circ} 14^{\prime} 39^{\prime \prime} \mathrm{N}, 7^{\circ} 19^{\prime} 28^{\prime \prime} \mathrm{E}, \mathrm{h}=985 \mathrm{~m}\right)$, Cuneo-S. Rocco ( $\left.4^{\circ} 21.4^{\prime} \mathrm{X}, 7^{\circ} 31.9^{\prime} \mathrm{E}, \mathrm{h}=597 \mathrm{~m}\right)$,

Roburent ( $\left.44^{\circ} 17^{\prime} 41.3^{\prime \prime} \mathrm{N}, 7^{\circ} 52^{\prime} 13.7^{\prime \prime} \mathrm{E}, \mathrm{h}=806 \mathrm{~m}\right)$.

Le prime due hanno iniziato la loro attiviti regolare nel NovembreDicembre 1966 e la terza nel Gennaio-Febbraio 1967.

Una serie di registrazioni sismografiche, estesa ad un rempo sufficientemente lungo, potrà, fra l'altro, permettere di localizzare i centri di scuotimento esistenti nella Valle del Gesso e nelle zone circostanti e di controllare la frequenza dei movimenti sismici, anche di piccola intensità, da essi originati.

\section{BIBIIOGRAFIA}

SACco F., La caverna ossifera del Bandito in Val Gesso. A cura del C.A.I. 'Torino, 1890.

Mrercaldi G., I terremoti della Liguria e del Piemonte. Napoli 1897.

SACCo F., I IIonti di Cuneo tra il Gruppo della Besimauda e quello dell'Argentera. R. Acc. delle Scienze di Torino (1906-1907).

SaCco F., Il Gruppo dell Argentera. R. Acc. delle Sc. di Torino, 1910-1911. Cavasino A., I terremoti d'Italia nel trentacinquennio 1599-1903. Roma, 1935. Rotí́ J. P., Les séismes des Alpes Frangaises en 19.38 et la séismicité des Alpes Occidentales. Ann. de l'Institut de Physique du Globe, Strasbourg, 1938 .

Rotiń J. P., La séismicité des Alpes Occidentales (Complements). Ann. de l'Inst. du Physique du Globe, Strasbourg, 1948.

Ascueri A., Geologia della media Valle del Gesso nella zona fra Taldieri, Entracque e Roaschia. Como, S.A.G.S.A., 1955.

Malaroda R. e Ramond C., Linee di dislocazione e sismiciti in Italia. "Boll. di Geodesia e Scienze Affini " dell Istituto Geografico Militare, 1957 11. 3.

DE PANFilis M., Attività sismica in Italia dal 19.5.3 al 19.5\%. "Anuali di Geofisica », XII, 1, 1959. 